\title{
Optimización del overrun (aireado), de la dureza, la viscosidad y los costos de un helado mediante el diseño de mezclas
}

\author{
Jorge Efraín Michue Mango* \\ Christian René Encina Zelada** \\ Fanny Emma Ludeña Urquizo*** \\ Universidad Nacional Agraria La Molina. Lima, Perú.
}

Recibido: 20 de mayo de 2015 / Aprobado: 21 de julio de 2015

\begin{abstract}
Resumen: El presente trabajo de investigación experimental tiene como objetivo principal utilizar el diseño de mezclas para optimizar cuatro factores en estudio: el overrun (aireado), la dureza, la viscosidad y los costos de un helado de crema, para lo cual la formulación que mejor cumplió con este proceso de optimización fue la que contenía 8,049 \% de crema de leche, $3,272 \%$ de manteca vegetal y $4,679 \%$ de polidextrosa.
\end{abstract}

Palabras clave: helado / diseño de mezclas / overrun / dureza / viscosidad / costos

\section{Optimization of Overrun, Hardness, Viscosity and Costs for Ice Cream Applying Mix Design}

\begin{abstract}
This research had as main principal aim use amix design to optimize four factors in studied: overrun, hardness, viscosity and cost of ice cream, for which the best formula fulfilled this optimization process was one containing $8,049 \%$ of cream, 3,272 \% of shortening and 4,679 \% polydextrose.
\end{abstract}

Key words: ice cream / design / mixing overrun / hardness / viscosity / costs

Correos electrónicos: *jmichue@lamolina.edu.pe, **cencina@lamolina.edu.pe, ***fludena@lamolina.edu.pe 


\section{INTRODUCCIÓN}

La elaboración de helados, tanto en el ámbito nacional como en el internacional, ha adquirido una importancia económica y social de singular importancia. Desde la antigüedad hasta nuestros días el consumo de helados ha experimentado cambios desde el punto de vista tecnológico, que ha posibilitado extender su consumo a prácticamente todas las clases sociales.

Los helados son aquellos productos alimenticios edulcorados, obtenidos a partir de una emulsión de grasa y proteínas, con la adición de otros ingredientes, o a partir de una mezcla de agua y otros componentes que se someten a congelación con o sin incorporación de aire, y se almacenan, distribuyen y expenden en estado de congelación o parcialmente congelados (Indecopi, 2006).

Según Madrid y Cenzano (2003), los helados de crema son aquellos cuyo ingrediente básico es la nata o crema de leche, por lo que su contenido en grasa de origen lácteo es más alto que en el resto de los otros tipos de helados. La nata, como se sabe, es un producto rico en materia grasa (18 a $55 \%$ ). Además de nata, este tipo de helado lleva azúcar, aire (que se incorpora durante el batido), espesantes, etc. El helado, producto de nuestro proyecto, pesará como mínimo 475 gramos por litro y su composición básica será:

- Azúcares. Una proporción mínima del 13 \%. De este total, la mitad deberá ser sacarosa o azúcar común, pudiendo el resto corresponder a otros, como glucosa, lactosa, etc.

- Grasa de leche. Tendrá $8 \%$ como mínimo. Según otras legislaciones, deberá ser de un $9 \%$ mínimo.

- Proteína láctea. En una proporción de 2,5 \% como mínimo.

- Extracto seco total. Contendrá 29 \% como mínimo. El extracto seco total es la cantidad de sólidos de un alimento. Es decir, es la suma de sus componentes (grasa, hidratos de carbono, proteínas, vitaminas, etc.).

Para Madrid y Cenzano (2003), los helados son una mezcla de diversos productos alimenticios, entre los que destacan: agua potable, leche y derivados lácteos (leche en polvo, crema de leche, leche fresca, suero de leche), azúcares diversos (sacarosa, glucosa, edulcorantes), grasas vegetales (coco, palma, etc.), frutas y zumos de fruta, etc. 
- Hidratos de carbono (azúcares). Son la fuente de energía de los seres vivos, que suministran lo necesario para nuestros movimientos. La glucosa se utiliza mucho en la fabricación de helados y sirve para bajar el punto al cual se produce la congelación de la mezcla (Madrid y Cenzano, 2003).

- Grasas. Compuestos de carbono, hidrógeno y oxígeno, con predominio del hidrógeno; en su combustión desprenden mayor número de calorías que los hidratos de carbono (Madrid y Cenzano, 2003).

- Sólidos no grasos lácteos (S.N.G.L). Timm, Hirsing, Buchner, Lips, y Geyer (1989) mencionan que los sólidos no grasos de leche son muy necesarios para que los helados obtengan una textura más firme y un cuerpo más cremoso y esponjoso, y con mayor volumen. Si se utiliza en poca cantidad se debilita la estructura del helado, si es en exceso lo vuelve arenoso, por lo que es fundamental para obtener un apropiado balance de sólidos en la mezcla. Los S.N.G.L están compuestos por proteínas (mayormente caseína), lactosa y sales minerales (calcio, potasio, fósforo, magnesio, hierro, etc.).

- Agua y aire. Timm et al. (1989) refieren que el helado debe su naturaleza al agua congelada, por ello esta es el componente más peculiar. El agua es responsable del carácter refrescante del producto, es el medio disolvente de los ingredientes hidrosolubles (azúcares, proteínas, sales, ácidos, sustancias aromáticas) y determina la consistencia del helado de acuerdo con la proporción congelada. En el helado el agua se encuentra repartida en forma de cristales de hielo en una matriz, que además contiene agua líquida. El número y las dimensiones de los cristales de hielo determinan esencialmente la consistencia del helado.

El desarrollo de cualquier nuevo producto alimenticio que involucre más de un ingrediente requiere alguna forma de experimento de mezcla, en contraposición a un experimento factorial. Este hecho es frecuentemente desconocido, por lo que se utilizan diseños experimentales inapropiados (Hare, 1974).

La teoría general del diseño óptimo se aplica a los experimentos de mezcla. Sin embargo, la restricción introduce algunas características especiales. En particular, los cambios en los valores de uno de los factores conducen a cambios en el valor de al menos uno de los otros factores. El diseño óptimo de experimentos se basa en una teoría que, como cualquier otra teoría general, proporciona una unificación de los 
muchos resultados por separado y una forma de la generación de nuevos resultados en situaciones nuevas (Atkinson, Dovev y Tobias, 2007).

La característica distintiva de un diseño de mezclas consiste en que los factores controlables o independientes representan cantidades proporcionales de la mezcla en vez de cantidades no circunscritas a ella; donde las proporciones son expresadas por volumen, por peso o por fracción molar. Si la suma de las proporciones de los componentes es menor que la unidad, por ejemplo, si la suma es igual a 0,80 porque el 0,20 de la mezcla se mantiene constante, y se desea trabajar solo con las proporciones variables que suman 0,80 , entonces dichas proporciones deben ser escritas como fracciones escaldadas, de tal forma que estas sumen la unidad (Cornell, 2002).

Según Cornell (2002), las principales consideraciones relacionadas con la exploración de la superficie de respuesta sobre la región simplex son:

- Elección de un modelo matemático apropiado, que describa aproximadamente la superficie sobre la región de interés.

- Evaluación de la suficiencia del modelo para representar la superficie de respuesta.

- Un diseño apropiado para recolectar las observaciones, ajustar el modelo y evaluar la suficiencia de ajuste.

En general, para representar $\varnothing(\mathrm{x} 1, \mathrm{x} 2, \ldots \mathrm{xq})$ se utilizan las funciones polinomiales. La justificación es que uno puede expandir $\varnothing$ $(\mathrm{x} 1, \mathrm{x} 2, \ldots \mathrm{xq})$ usando una serie de Taylor $\mathrm{y}$, por ende, puede utilizarse un polinomio con una aproximación (Cornell, 2002).

Normalmente, un polinomio de bajo grado, tal como el de primer grado

$$
\eta=\beta_{0}+\sum_{i=1}^{q} \beta_{i} X_{i}
$$

o el de segundo grado

$$
\eta=\beta_{0}+\sum_{i=1}^{q} \beta_{i} X_{i}+\sum_{i}^{q} \sum_{\leq j}^{q} \beta_{i j} X_{i} X_{j}
$$


es el tipo de modelo adecuado para representar la superficie. Las ecuaciones polinómicas de bajo grado son más convenientes de manejar en comparación con las ecuaciones de grados más altos, debido a que los polinomios de más bajo grado contienen pocos términos, y por ello requieren pocos valores observados de la respuesta para estimar los parámetros (los B’s) de la ecuación (Cornell, 2002).

El objetivo principal del presente trabajo de investigación fue realizar la optimización del overrun (aireado), de la dureza, de la viscosidad y de los costos de un helado con crema de leche, manteca vegetal y polidextrosa, mediante el diseño de mezclas.

\section{PARTE EXPERIMENTAL}

\subsection{Lugar de ejecución}

El presente trabajo de investigación se realizó en la Planta Piloto de Leche y Laboratorio de Investigación en Leche y Productos Lácteos de la Universidad Nacional Agraria La Molina (UNALM) y en el Laboratorio de Análisis Físico-Químico de Alimentos y Laboratorio de Investigación e Instrumentación de la Facultad de Industrias Alimentarias de la misma universidad.

\subsection{Materiales y reactivos}

Los materiales y reactivos utilizados en la investigación se detallan a continuación:

- Materias primas y aditivos alimentarios: leche descremada en polvo; manteca vegetal (tropical); agua; azúcar blanca; suero de leche en polvo; crema de leche ( $35 \%$ de grasa); dextrosa; agua de mesa; cremodan SB 803 (mezcla de estabilizantes y emulsificantes) proporcionado por Danisco (Estados Unidos) y esencia de vainilla.

- Equipos para la elaboración del helado: balanza analítica Mettler, capacidad de 160 g, con precisión de 0,0001 g; balanza técnica Sauter (capacidad de $1 \mathrm{~kg}$, precisión de $0,1 \mathrm{~g}$ ); ollas de acero inoxidable (capacidades de $10 \mathrm{~L}, 20 \mathrm{~L}$ y $7 \mathrm{~L}$ ); espátula de acero inoxidable; mesa de acero inoxidable; baldes de plástico, capacidad de $8 \mathrm{~L}$; recipientes de plástico; cocina eléctrica marca Teba; cocina de gas; termóme- 
tro digital de rango -50 a $200{ }^{\circ} \mathrm{C}$, marca Thermo-Pro (con sensor de acero inoxidable terminal en punta); homogeneizador marca Rannie, modelo LAB.MB serie N. ${ }^{\circ} 3722$; cámara de refrigeración de la Planta de Leche de la UNALM (temperatura promedio $3 \pm 1^{\circ} \mathrm{C}$ ); congeladora marca Frigidaire, modelo AFFCm; batidora para helados con sistema de enfriamiento y superficie rascada, modelo A 424, serie N. ${ }^{\circ} 41264$ GEN EQUIP MFG.Co, INDPLS.IND.

- Equipos y materiales para el análisis del helado: texturómetro Brookfield-Farnell QTS-25; reómetro Brookfield DV-II Ultra, modelo RVDV-II Ultra, serie N. ${ }^{\circ} 482670$; programa estadístico DesingExpert 9.0.4.1.

\subsection{Métodos de análisis}

Los métodos de análisis son los siguientes:

- Determinación de la viscosidad: la viscosidad se midió siguiendo la metodología descrita por Alfaifi y Stathopoulos (2010). Se utilizó el reómetro de Brookfield (modelo DV-II, spindle \#2) a 50 rpm y 5 segundos para determinar la viscosidad; las muestras se tomaron después del madurado y antes del batido en un beaker de $200 \mathrm{ml}$ a una temperatura de $4 \pm 0,5^{\circ} \mathrm{C}$, los resultados se midieron en Centipoises $(\mathrm{cP})$, valor que equivale a $\mathrm{g} \cdot(\mathrm{s} \cdot \mathrm{cm})^{-1}$.

- Determinación del aireado (overrun): se determinó utilizando el método descrito por Datta, Sahin, Sumnu y Keshin (2007) y AOAC (1988), aplicado por Alfaifi y Stathopoulos (2010). La medición del aireado se tomó por muestra, comparando el peso de la mezcla base y el helado en un recipiente de volumen fijo, de acuerdo con la siguiente ecuación:

$$
\text { Aireado }(\%)=\frac{\text { vol. helado }- \text { vol. mezcla }}{\text { vol. mezcla }} \times 100
$$

Los resultados se expresan en porcentaje de aire incorporado luego del batido con respecto a la mezcla base; la temperatura de la base será de $4{ }^{\circ} \mathrm{C}$ y del helado a la salida del batidor de $-5^{\circ} \mathrm{C}$.

- Determinación de la dureza: se determinó usando el método de Roland, Phillips y Book (1999), aplicado por Lim et al. (2008). La dureza se determinó en el laboratorio a temperatura ambiente de $25 \pm 2{ }^{\circ} \mathrm{C}$. Se utilizó un texturómetro QTS25 equipado con un cilindro acrílico de 
$2,5 \mathrm{~cm}$ de diámetro. La velocidad de compresión fue de $2 \mathrm{~mm} / \mathrm{s}$ hasta una distancia de $20 \mathrm{~mm}$; las muestras se tomaron a la salida de la batidora, en envases de plástico de $6 \mathrm{~cm}$ de diámetro y $3 \mathrm{~cm}$ de altura, se almacenó a $-18^{\circ} \mathrm{C}$, los resultados se expresaron en gramos.

- Determinación del costo por insumos: el costo por insumos de la elaboración de helados se ve influenciado por el costo individual de los ingredientes. En la elaboración de helado con crema de leche se tiene un costo alto, por eso se evaluó la mezcla de crema de leche, manteca vegetal y polidextrosa, teniendo en cuenta los niveles de los componentes. Los datos se procesaron en una hoja de cálculo.

\subsection{Metodología experimental}

En la primera etapa de la investigación se planteó el flujo de operaciones para la elaboración de helados de crema, el cual fue necesario para estandarizar el proceso y posterior aplicación de las formulaciones para el diseño de mezclas.

En la segunda etapa de la investigación se realizó la determinación del polinomio canónico de Scheffé, necesario para poder realizar el modelamiento matemático de los tres componentes principales o factores en estudio (crema de leche, manteca vegetal y polidextrosa) en función a las variables respuestas planteadas para esta investigación (overrun, viscosidad, dureza y costos). Se establecieron los rangos mínimos y máximos de los tres componentes (ingredientes) principales para el presente trabajo de investigación, que fueron: la crema de leche, la manteca vegetal y la dextrosa; valores que se obtuvieron como resultado de realizar pruebas preliminares, y de trabajos previos vistos en papers y tesis relacionadas con el tema de investigación presentado.

La metodología experimental se realizó en base a la formulación base presentada anteriormente en la tabla 1 y tuvo como finalidad evaluar el efecto simultáneo de la mezcla de los ingredientes antes mencionados sobre el aireado (overrun), la viscosidad, la dureza y los costos. Se consideró la metodología propuesta por Cornell (2002) para el caso de 3 ingredientes aplicando el Diseño de Mezclas (Design Expert, 2015).

Finalmente, y después de obtenidos los modelos matemáticos para cada una de las respuestas estudiadas, se procedió a la optimización simultánea de estas. Para ello se tuvo en cuenta la metodología de la deseabilidad global, descrita por Cornell (2002). El objetivo de esta optimización fue encontrar aquella mezcla que cumpliera de la mejor 
forma con determinadas restricciones, impuestas sobre cada una de las respuestas.

Para realizar la optimización de las respuestas se procedió a utilizar las características del helado de crema y sus restricciones de overrun, dureza, viscosidad y costos; de esta manera, la formulación seleccionada como óptima debía contar con las siguientes características:

- overrun: mayor es mejor

- dureza: menor es mejor

- viscosidad: menor es mejor

- costo de producción: menor es mejor

Tabla 1

Fórmula base para elaboración del helado

\begin{tabular}{lc}
\hline Insumos & Porcentajes \\
\hline Crema de leche & 8 \\
Manteca vegetal & 2 \\
Polidextrosa & 6 \\
Suero de leche & 1 \\
Leche en polvo descremada & 9 \\
Agua & 58,98 \\
Azúcar & 14,67 \\
Estabilizante & 0,35 \\
Total & 100 \\
\hline
\end{tabular}

Elaboración propia

Tabla 2

Restricciones sobre tres ingredientes para elaborar helados aplicando el diseño de mezclas

Límites en función de fórmula total (\%)

$$
\begin{gathered}
6 \leq \text { Crema de leche } \leq 10 \\
1 \leq \text { Manteca vegetal } \leq 5 \\
1 \leq \text { Polidextrosa } \leq 9
\end{gathered}
$$

\footnotetext{
Elaboración propia
} 


\subsubsection{Análisis estadístico}

Con los tres componentes o ingredientes: crema de leche, manteca vegetal y polidextrosa y sus respectivos límites, se realizó el diseño de mezclas utilizando el programa estadístico Design-Expert ${ }^{\circledR}$ 9.0.4.1; y se evaluó el efecto de esos tres componentes sobre las características o variables respuestas analizadas: overrun, viscosidad, dureza y costos. Se realizó un análisis de regresión múltiple (métodos de mínimos cuadrados), con la finalidad de obtener el polinomio canónico de Scheffé que describe la dependencia de dicha característica en función de los ingredientes bajo estudio (Cornell, 2002). La determinación de dicho polinomio, así como su respectivo análisis de varianza (ANVA) y prueba de significancia de coeficientes (nivel de significación $\alpha=0.05$ ), fueron llevados a cabo mediante la utilización del programa estadístico Design-Expert ${ }^{\circledR}$ 9.0.4.1.

\section{RESULTADOS Y DISCUSIÓN}

\subsection{Operaciones para la elaboración de helados de crema}

En la figura 1 se detallan todos los procesos unitarios aplicados para la elaboración de las fórmulas de helado de crema. A continuación se explican cada uno de esos procesos:

- Recepción, almacenado y pesado. Se recibieron las materias primas bajo un control sensorial y fisicoquímico y aplicando en todo momento las buenas prácticas de manipulación de alimentos (BPM), luego se procede a colocarlos en los almacenes respectivos, los ingredientes se pesan de acuerdo con la formulación establecida.

- Mezclado 1. Se procedió a calentar el agua en una olla hasta $30^{\circ} \mathrm{C}$, enseguida se disolvió los ingredientes en polvo, a los $45^{\circ} \mathrm{C}$ se disolvió el estabilizante mezclado con una parte de azúcar, a $60^{\circ} \mathrm{C}$ se adicionó la otra parte de azúcar con polidextrosa, luego la crema de leche y manteca vegetal, en todo momento se mantuvo una agitación constante, hasta llegar a $80^{\circ} \mathrm{C}$.

- Pasteurizado. Constituye un proceso fundamental para garantizar la calidad microbiológica del producto. Consiste en calentar en una olla rápidamente la mezcla a $80^{\circ} \mathrm{C}$ por 10 minutos y enseguida enfriar con rapidez hasta $4{ }^{\circ} \mathrm{C}$. El objetivo es destruir bacterias patógenas, facilitar la disolución de ingredientes, activar los estabilizantes y emulsificantes, mejorar el sabor y aumentar el tiempo de conservación. 


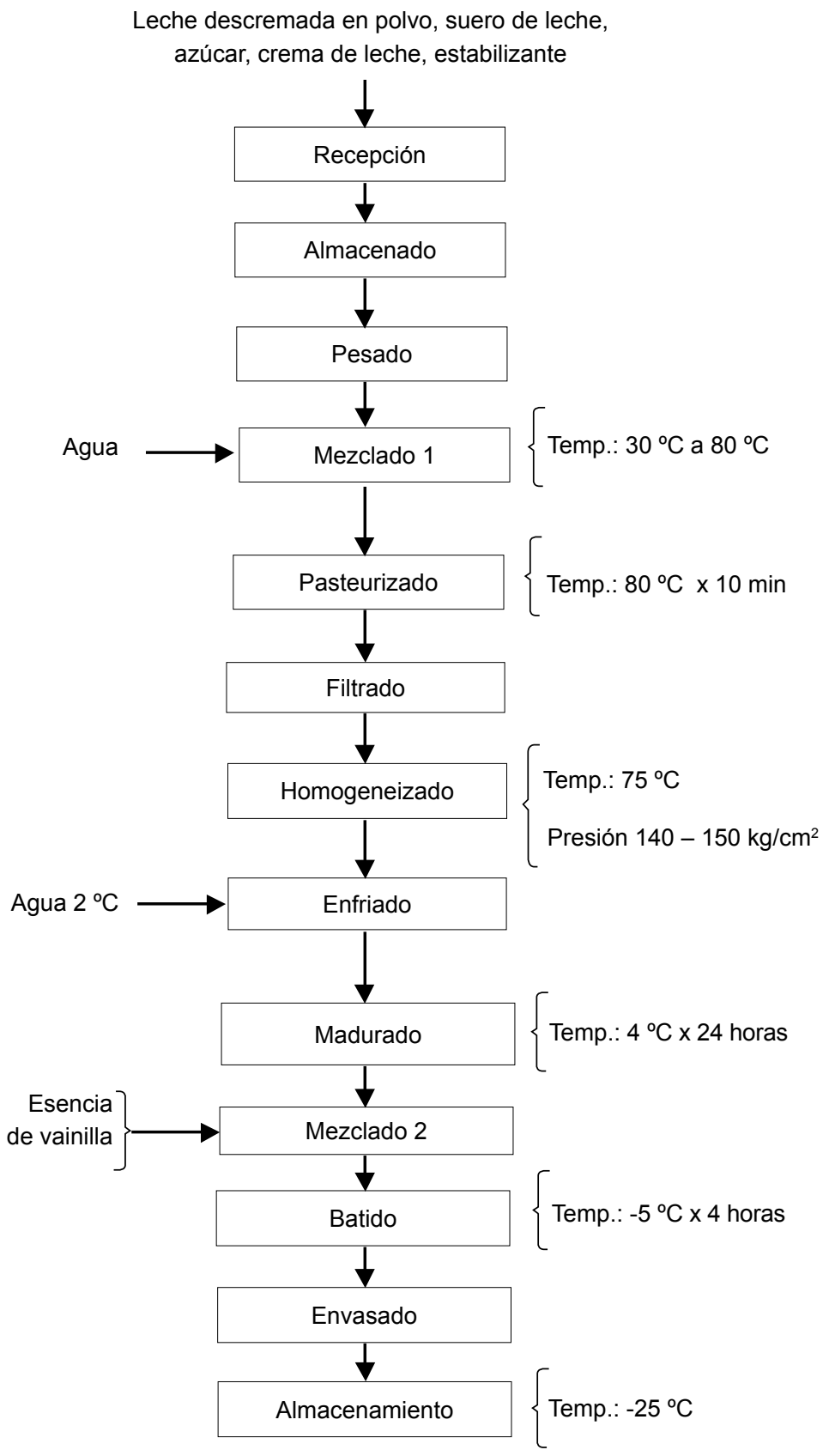

Figura 1. Flujo de operaciones para la elaboración de helados de crema Elaboración propia 
- Homogeneización. Este proceso fue realizado mediante el paso forzado de la mezcla a través de un pequeño orificio bajo las condiciones de temperatura $75{ }^{\circ} \mathrm{C}$ y entre 140 a $150 \mathrm{~kg} / \mathrm{cm}^{2}$ de presión determinadas empleando una bomba de desplazamiento positivo. La homogeneización se realizó en un homogeneizador marca Rannie modelo LAB.MB serie N. ${ }^{\circ} 3722$.

- Enfriamiento. El enfriamiento se realizó a la temperatura de $4{ }^{\circ} \mathrm{C}$, con el fin de retardar el crecimiento de microorganismos; asimismo, la grasa se cristaliza, esto hace que se ionice para que pueda enlazarse con los estabilizantes y las proteínas en un tiempo de media hora (Fraser, 1998).

- Maduración. Durante esta etapa la grasa se solidificó, las proteínas y los estabilizantes se hidrataron, aumentó la viscosidad, mejorando la suavidad y el cuerpo del helado, y mostró mayor resistencia al derretimiento. La maduración se realizó en un balde almacenado a $4{ }^{\circ} \mathrm{C}$ en la cámara de refrigeración por 24 horas (Brennan, Butters y Cowell, 1998).

- Mezclado 2. Se procedió a mezclar la esencia de vainilla mediante agitación manual constante, hasta alcanzar una mezcla uniforme.

- Congelamiento e incorporación de aire (batido). Es una de las operaciones más importantes en la fabricación de helado, de ella depende la calidad, la palatabilidad y el rendimiento. La mezcla fue rápidamente congelada mientras era agitada, lográndose así la incorporación de aire y la formación de pequeños cristales de hielo, necesarios para las características de suavidad, palatabilidad y aireado adecuado del producto final; el batido se realizó en un intercambiador de calor de superficie raspada, la temperatura de salida es de $-5{ }^{\circ} \mathrm{C}$ en un tiempo de 15 minutos.

- Envasado. Se envasó en recipientes rígidos de polietileno de $6 \mathrm{~cm}$ de diámetro y $3 \mathrm{~cm}$ de altura, en un 1 minuto.

- Almacenamiento. Una vez que el helado alcanzó su temperatura de conservación $\left(-25^{\circ} \mathrm{C}\right)$ en la congeladora, se encontró listo para ser analizado (según las diferentes formulaciones) en las siguientes etapas de la investigación. 


\subsection{Determinación del polinomio canónico de Scheffé}

Los valores observados del overrun, de la dureza, la viscosidad y los costos correspondientes a cada una de las formulaciones de helado estudiadas, son presentados en la tabla 3; asimismo, la representación gráfica de estos valores son presentados en las figuras $2,3,4$ y 5.

Tabla 3

Resultados obtenidos del overrun (\%), de la dureza ( $\mathrm{kg}$ ), la viscosidad (cP) y los costos (S/.) de las 20 formulaciones analizadas

\begin{tabular}{cccccccc}
\hline Fórmula & $\begin{array}{c}\text { Manteca } \\
\text { vegetal (\%) }\end{array}$ & $\begin{array}{c}\text { Crema } \\
\text { de leche } \\
(\%)\end{array}$ & $\begin{array}{c}\text { Polidex- } \\
\text { trosa (\%) }\end{array}$ & $\begin{array}{c}\text { Overrun } \\
\mathbf{( \% )}\end{array}$ & $\begin{array}{c}\text { Dureza } \\
\mathbf{( k g )}\end{array}$ & $\begin{array}{c}\text { Viscosidad } \\
\text { (cP) }\end{array}$ & $\begin{array}{c}\text { Costo } \\
\text { (S/.) }\end{array}$ \\
\hline F1 & 10,00 & 3,06 & 2,94 & 43,00 & 18976.67 & 433.87 & 24,59 \\
F2 & 6,00 & 2,84 & 7,16 & 47,35 & 14982.33 & 539.95 & 26,96 \\
F3 & 8,00 & 3,00 & 5,00 & 46,48 & 13428.67 & 552.27 & 25,72 \\
F4 & 6,00 & 1,00 & 9,00 & 46,29 & 11760.33 & 502.60 & 28,70 \\
F5 & 10,00 & 5,00 & 1,00 & 42,97 & 25530.67 & 637.66 & 22,75 \\
F6 & 6,00 & 5,00 & 5,00 & 46,23 & 21076.67 & 567.47 & 24,91 \\
F7 & 8,68 & 1,48 & 5,84 & 46,97 & 14158.67 & 443.52 & 26,80 \\
F8 & 6,00 & 1,00 & 9,00 & 46,45 & 11886.33 & 504.20 & 28,70 \\
F9 & 10,00 & 1,00 & 5,00 & 45,25 & 11873.33 & 428.75 & 26,54 \\
F10 & 7,30 & 4,43 & 4,27 & 51,69 & 20740.33 & 507.25 & 24,75 \\
F11 & 6,66 & 3,66 & 5,68 & 48,46 & 14535.67 & 561.87 & 25,82 \\
F12 & 8,00 & 3,00 & 5,00 & 46,58 & 13450.67 & 550.51 & 25,72 \\
F13 & 7,82 & 1,00 & 7,18 & 47,11 & 12506.67 & 449.36 & 27,72 \\
F14 & 8,01 & 5,00 & 2,99 & 48,55 & 20715.33 & 526.08 & 23,82 \\
F15 & 9,37 & 2,35 & 4,28 & 45,36 & 12509.00 & 459.79 & 25,60 \\
F16 & 6,00 & 5,00 & 5,00 & 46,32 & 21027.67 & 565.81 & 24,91 \\
F17 & 8,00 & 3,00 & 5,00 & 46,64 & 13442.67 & 550.88 & 25,72 \\
F18 & 6,00 & 2,84 & 7,16 & 47,23 & 15050.33 & 539.52 & 26,96 \\
F19 & 8,69 & 3,68 & 3,63 & 44,31 & 19098.00 & 453.33 & 24,71 \\
F20 & 10,00 & 5,00 & 1,00 & 42,93 & 25532.67 & 639.40 & 22,75 \\
\hline & & & & & & & \\
\hline
\end{tabular}

Elaboración propia 
Para el overrun, después de someter los valores observados (tabla 3) al análisis de regresión múltiple correspondiente, se obtuvo la siguiente ecuación polinomial de primer grado (ecuación 3):

$$
\begin{aligned}
\hat{\mathrm{y}}= & 0.39978 x_{1}+3.06827 x_{2}+0.81760 x_{3}+0.34647 x_{1} x_{2}+ \\
& 0.60264 x_{1} x_{3}-0.1068 x_{2} x_{3}
\end{aligned}
$$

Donde $\hat{y}$ representa el overrun del helado estimado o predicho (\%); mientras que $x_{1}, x_{2}$ y $x_{3}$ corresponden a los porcentajes de manteca vegetal, crema de leche y polidextrosa, respectivamente, presentes en el helado. La representación gráfica de esta tendencia se observa en la figura 2 .

Para la dureza, después de someter los valores observados (tabla 3) al análisis de regresión múltiple correspondiente, se obtuvo la siguiente ecuación polinomial de primer grado (ecuación 4):

$$
\begin{aligned}
\hat{\mathrm{y}}= & 2045.00288 x_{1}+7880.49279 x_{2}+2133.31204 x_{3} \\
& -607.14531 x_{1} x_{2}-348.36447 x_{1} x_{3}-507.53025 x_{2} x_{3}
\end{aligned}
$$

Donde $\hat{y}$ representa la dureza del helado estimada o predicha (\%); mientras que $x_{1}, x_{2}$ y $x_{3}$ corresponden a los porcentajes de manteca vegetal, crema de leche y polidextrosa, respectivamente, presentes en el helado. La representación gráfica de esta tendencia se observa en la figura 3.

Para la viscosidad, después de someter los valores observados (tabla 3) al análisis de regresión múltiple correspondiente, se obtuvo la siguiente ecuación polinomial de primer grado (ecuación 5):

$$
\begin{aligned}
\hat{\mathrm{y}}= & 21.7793 x_{1}+16.41429 x_{2}+60.40227 x_{3}+5.67357 x_{1} x_{2} \\
& -3.74631 x_{1} x_{3}-0.43229 x_{2} x_{3}
\end{aligned}
$$

Donde $\hat{y}$ representa la viscosidad del helado estimada o predicha (\%); mientras que $x_{1}, x_{2}$ y $x_{3}$ corresponden a los porcentajes de manteca vegetal, crema de leche y polidextrosa, respectivamente, presentes en el helado. La representación gráfica de esta tendencia se observa en la figura 4. 
Para los costos, después de someter los valores observados (tabla 3) al análisis de regresión múltiple correspondiente, se obtuvo la siguiente ecuación polinomial de primer grado (ecuación 6):

$$
\hat{\mathrm{y}}=1.51556 x_{1}+1.10758 x_{2}+2.05555 x_{3}
$$

Donde $\hat{y}$ representa la viscosidad del helado estimada o predicha (\%); mientras que $x_{1}, x_{2}$ y $x_{3}$ corresponden a los porcentajes de manteca vegetal, crema de leche y polidextrosa, respectivamente, presentes en el helado. La representación gráfica de esta tendencia se observa en la figura 5.

Tabla 4

Prueba de significancia para los coeficientes del modelo de regresión de las cuatro variables respuestas en la elaboración de helado

\begin{tabular}{llccc}
\hline Factor & Término de modelo & R cuadrado & Valor $\mathbf{F}$ & $\begin{array}{c}\text { p value } \\
\text { Prob }>\text { F }\end{array}$ \\
\hline Overrun & Cuadrático & 0.6585 & 536.53 & $<0.0001$ \\
Dureza & Cuadrático & 0.9269 & 1835.58 & $<0.0001$ \\
Viscosidad & Cuadrático & 0.6650 & 3083.60 & $<0.0001$ \\
Costos & Lineal & 1.0000 & 36.63 & $<0.0001$ \\
\hline
\end{tabular}

Elaboración propia

El resumen de los resultados del análisis de varianza ANVA de los modelos para los cuatro factores analizados: overrun, dureza, viscosidad y costos se muestran en la tabla 4. Como se puede apreciar, el valor $\mathrm{F}$ calculado es alto en todos los casos, en comparación con el valor tabular F, lo cual implica que el modelo es significativo. Con los valores obtenidos en el análisis de los coeficientes del modelo estimado, se puede afirmar que los modelos lineales fueron altamente significativos al 95, puesto que su valor $\mathrm{p}$ ( $p$ value) es inferior en todos los casos a 0.0001 . De acuerdo al coeficiente de determinación ( $R$ cuadrado), indica el valor de la variación en la respuesta que es explicada por el modelo seleccionado, que en todos los casos es mayor al 0,50 o $50 \%$. 

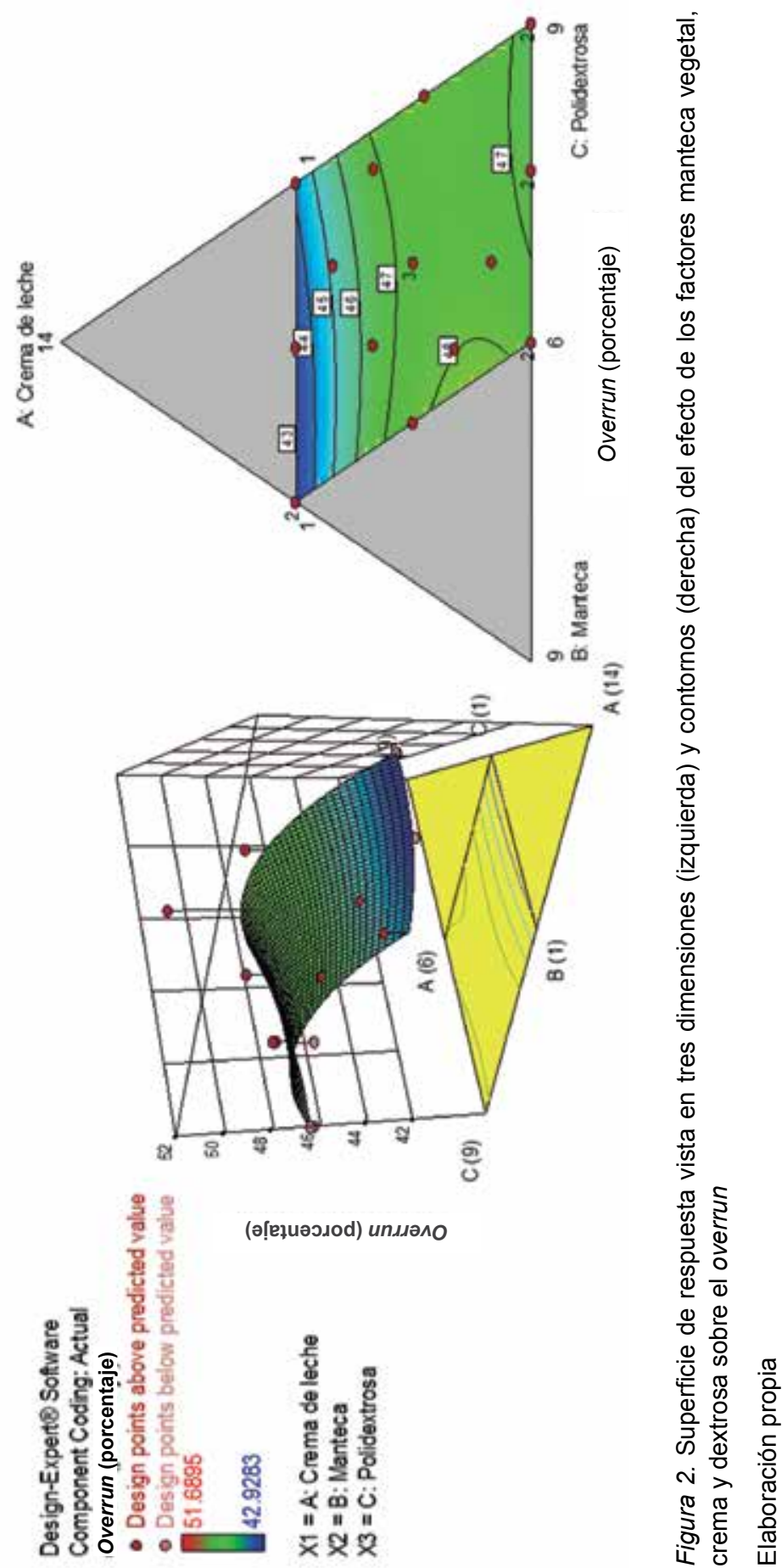

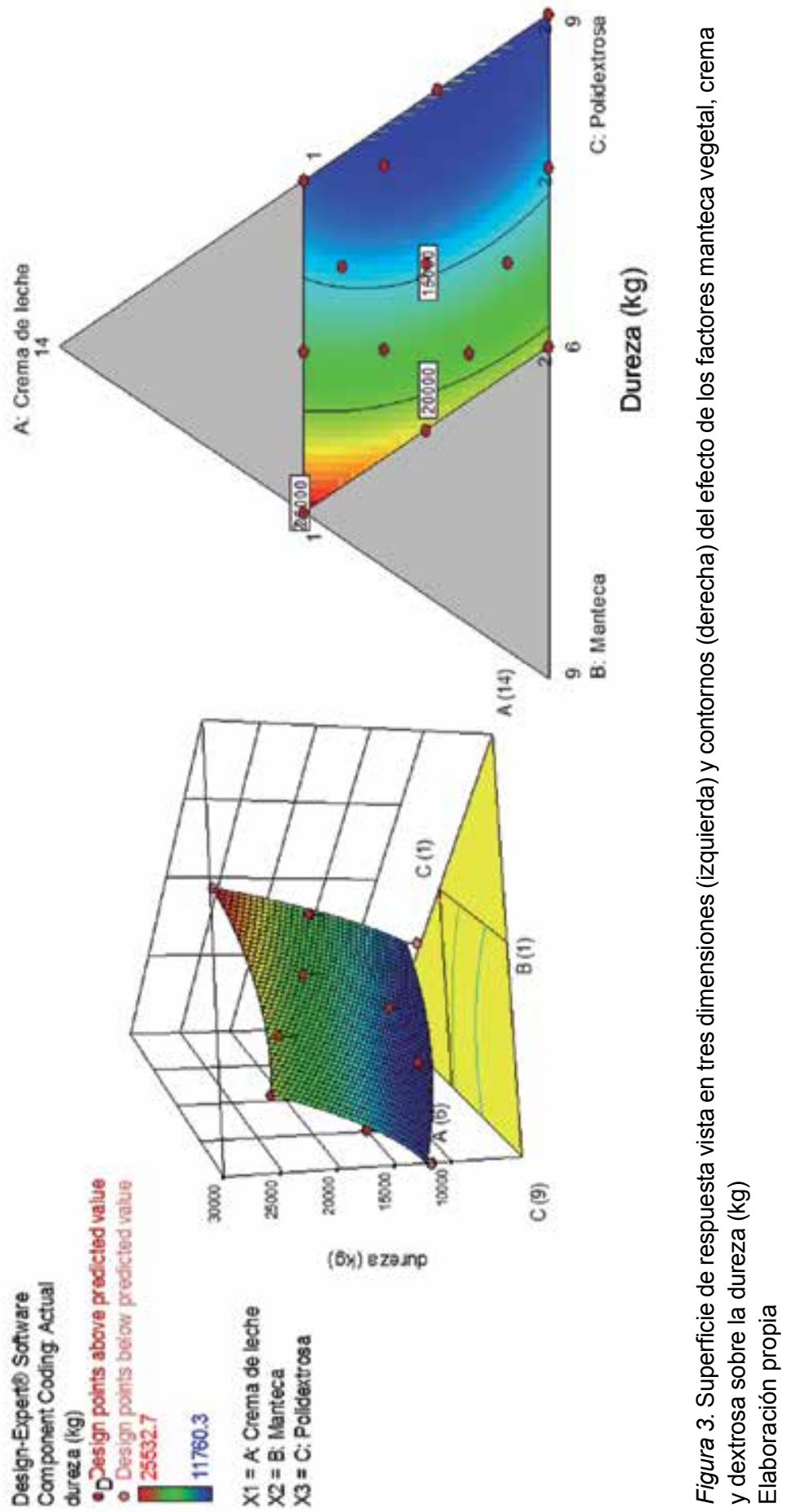


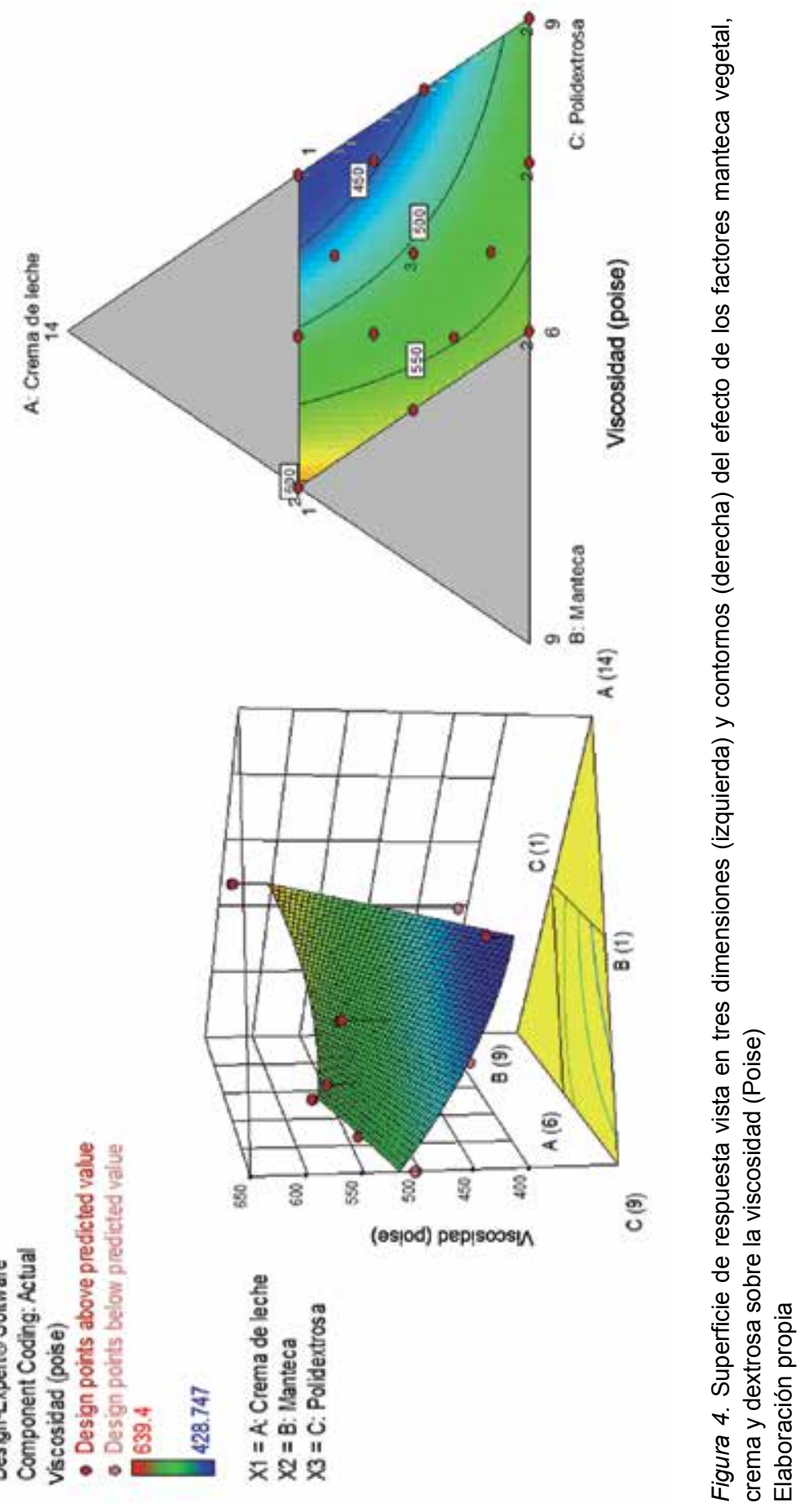




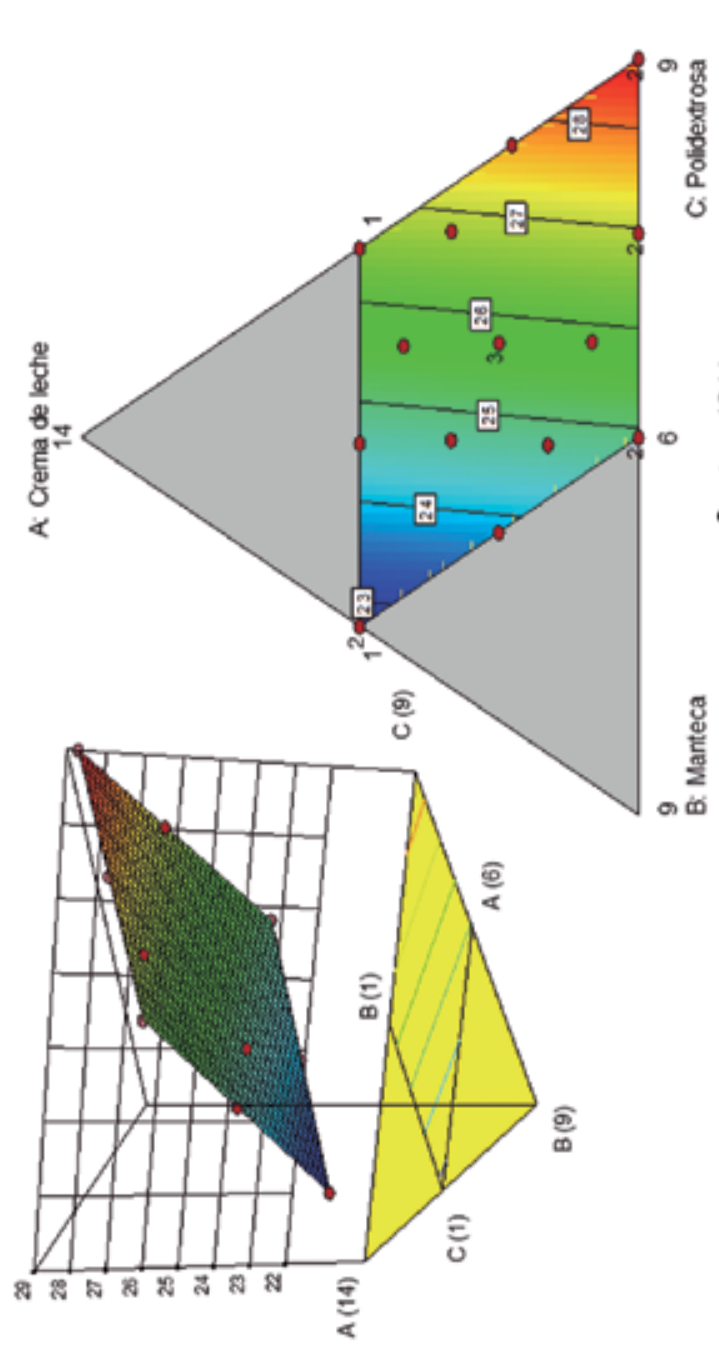

(is) 501500
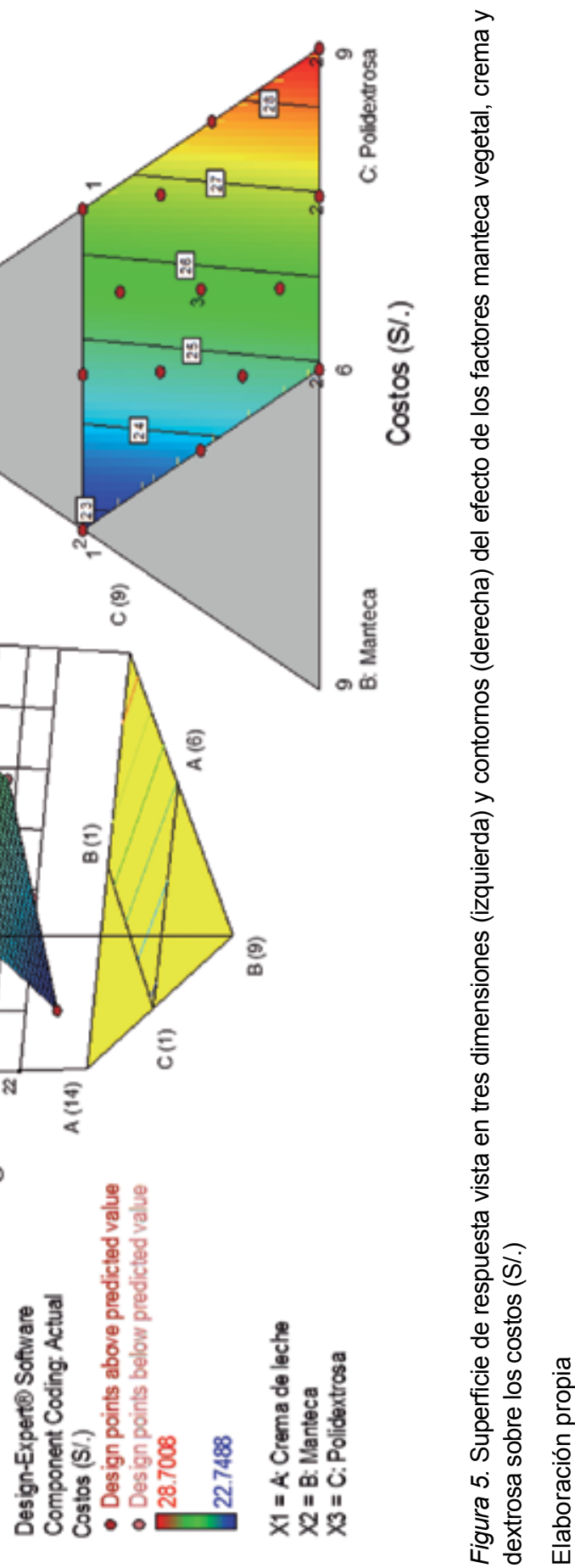


\subsection{Optimización simultánea de respuestas}

Después de obtenidos los modelos matemáticos para cada una de las respuestas estudiadas (ecuaciones $3,4,5$ y 6), se realizó la optimización simultánea de estas, de acuerdo con la metodología descrita por Cornell (2002); asignando un valor de 1 para el exponente $t$ en la función de deseabilidad. Para realizar la optimización de las respuestas, se procedió a utilizar las características del helado de crema y sus restricciones de overrun, dureza, viscosidad y costos, estas características son presentadas a continuación; de esta manera, la formulación seleccionada como óptima debía contar con las siguientes características:

- overrun: mayor es mejor; entre 42.9283 y 51.6895

- dureza: menor es mejor; entre 11760.3 y 25532.7

- viscosidad: menor es mejor; entre 428.747 y 639.4

- costo de producción: menor es mejor; entre 22.7488 y 28.7008

En la tabla 5 se indican las dos formulaciones óptimas que cumplen con las restricciones antes mencionadas, se puede apreciar el cálculo de deseabilidad global para cada formulación, en donde la mezcla denominada formulación 1 presenta el valor de deseabilidad global más elevado (0,568 o 56,8 \%), se aprecia un menor porcentaje de crema de leche, un mayor porcentaje de manteca vegetal y una menor proporción de polidextrosa en comparación con la fórmula 2 .

Por otro lado, la mezcla de formulación 2 , en comparación con la formulación 1, presentó una menor deseabilidad global (0,518 o 51,8 \%); se aprecia un mayor porcentaje de crema de leche, un menor porcentaje de manteca vegetal y una mayor proporción de polidextrosa. Debido a ello, comparándola con la formulación 1, el overrun (\%), dureza (kg), viscosidad (cP) disminuyen para los tres factores, mientras que el costo (S/.) aumenta.

Además, la figura 6 permite visualizar en el triángulo simplex el punto óptimo de formulación (mayor deseabilidad), el cual se obtuvo a partir de las restricciones impuestas sobre las respuestas (overrun, dureza, viscosidad y costos); a su vez, en la figura 4 se aprecia el punto a una mejor escala dentro del triángulo simplex. 
Tabla 5

Composición y características de las mezclas obtenidas tras la optimización simultánea de respuestas

\begin{tabular}{|c|c|c|c|c|c|c|c|c|}
\hline \multirow[b]{2}{*}{$\begin{array}{l}\text { Formu- } \\
\text { lación }\end{array}$} & \multicolumn{3}{|c|}{ Composición } & \multicolumn{4}{|c|}{ Características } & \multirow{2}{*}{$\begin{array}{c}\text { Desea- } \\
\text { bilidad } \\
\text { global } \\
\text { (D) }\end{array}$} \\
\hline & $\begin{array}{c}\text { Crema } \\
\text { de } \\
\text { leche }\end{array}$ & Manteca & $\begin{array}{l}\text { Polidex- } \\
\text { trosa }\end{array}$ & $\begin{array}{c}\text { Overrun } \\
(\%)\end{array}$ & Dureza (kg) & $\begin{array}{c}\text { Visco- } \\
\text { sidad } \\
\text { (cP) }\end{array}$ & $\begin{array}{c}\text { Costo } \\
\text { (S/. / } \\
100 \mathrm{~g})\end{array}$ & \\
\hline $\begin{array}{c}\text { Formulación } \\
1\end{array}$ & 8.049 & 3.272 & 4.679 & 47,269 & 15347.527 & 513.347 & 25.440 & 0.568 \\
\hline $\begin{array}{c}\text { Formulación } \\
2\end{array}$ & 8.876 & 1.000 & 6.124 & 46,803 & 11663.044 & 423.712 & 27.148 & 0.518 \\
\hline
\end{tabular}

Elaboración propia
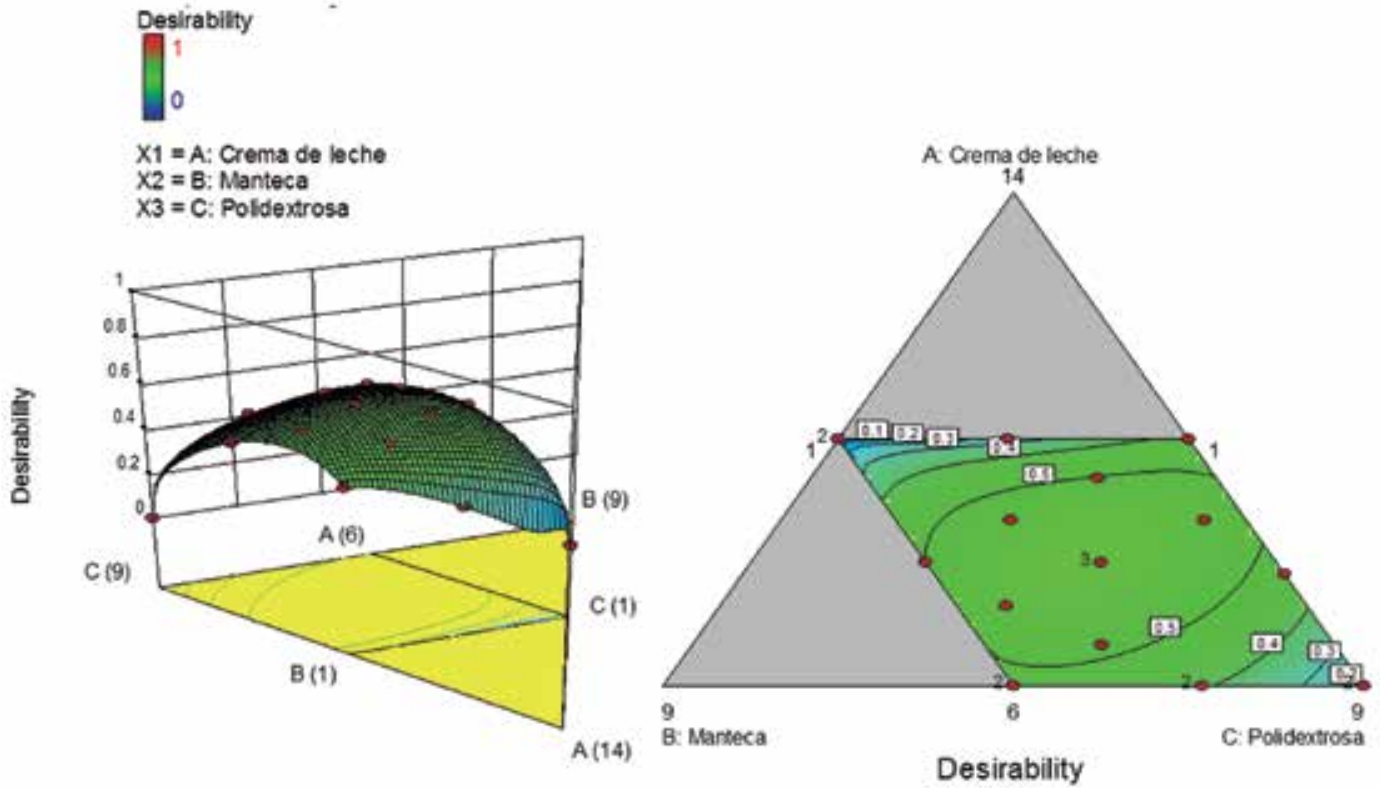

Figura 6. Superficie de respuestas múltiples visualizando el valor de deseabilidad (desirability) para los factores crema de leche, manteca vegetal y polidextrosa

Elaboración propia 


\section{CONCLUSIONES}

- La mejor fórmula de optimización simultánea tuvo un valor de deseabilidad global más elevado (0,568 o 56,8 \%), se apreció un menor porcentaje de crema de leche, un mayor porcentaje de manteca vegetal y una menor proporción de polidextrosa en comparación con la segunda mejor fórmula, la cual tuvo un contenido de 8,049 \% de crema de leche, 3,272 \% de manteca vegetal y 4,679\% de polidextrosa.

- La segunda mejor fórmula, en comparación con la mejor, presentó una menor deseabilidad global (0,518 o 51,8 \%); se apreció un mayor porcentaje de crema de leche, un menor porcentaje de manteca vegetal y una mayor proporción de polidextrosa; debido a ello, comparándola con la mejor fórmula, el overrun (\%), dureza (kg), viscosidad (cP) disminuyen para los tres factores, mientras que el costo (S/.) aumentó. Esta fórmula tuvo un contenido de 8,876 \% de crema de leche, $1,0 \%$ de manteca vegetal y $6,124 \%$ de polidextrosa.

\section{REFERENCIAS}

Alfaifi, M. S., y Stathopoulos, C. E. (2010). Effect of egg yolk substitution by sweet whey protein concentrate (WPC), on physical properties of gelato ice cream. International Food Research Journal 17, 787-793.

AOAC - Association of Official Analytical Chemist. (1988). Official methods of nalysis. (14. ${ }^{\mathrm{a}}$ ed.). Arlington, VA, Estados Unidos.

Atkinson, A., Donev, A., y Tobias, R. (2007). Optimum experimental designs, with SAS, 221-247. New York: Oxford University Press.

Brennan, J. G., Butters, J. R., y Cowell, N. D. (1998). Las operaciones de la ingeniería de los alimentos. Zaragoza, España: Acribia.

Cornell, J. (2002). Experiments with mixtures: designs models and the analysis of mixture data. (3. ${ }^{a}$ ed.). New York: John Wiley \& Sons, Inc.

Datta, A., Sahin, S., Sumnu, G., y Keskin, S. (2007). Porous media characterization of breads baked using novel heating modes. Journal of Food Engineering, 79, 106-116.

Desing Expert. (2015). User's guide version 7.16 for windows. Minneapolis, Estados Unidos: Stat-Eare, Inc.

Fraser, B. (1988). Manual de tecnología de los helados. Valdivia, Chile: Universidad Austral de Chile. 
Hare, L. (1974). Mixture designs applied to food formulations. Food Technology. 28(3), 1150-1162.

Indecopi - Instituto Nacional de Defensa de la Competencia y de la Protección de la Propiedad Intelectual. (2006). NTP 202.057:2006. Leche y productos lácteos. Helados: Requisitos. (2. ${ }^{\mathrm{a}}$ ed.). Lima: Indecopi.

Lim, S. Y., Swamson, B. G., Ross, C. F., y Clarks. (2008). Hydrostatic pressure modification of whey protein concentrate for improved bodu and texture of low fat ice cream. Journal of Dairy Science, 4(91), 1297-1307.

Madrid, A., y Cenzano, I. (2003). Tecnología de la elaboración de helados. Madrid: Coedición: A. Madrid Vicente, Ediciones/Mundi-Prens.

Roland, A., Phillips, L., y Boor, K. (1999). Effects of fat content on the sensory properties, melting, color and hardness of ice cream. Journal of Dairy Science, 82(1), 32-38.

Timm, F., Hirsing, I. Buchner, H., Lips, P., y Geyer, J. (1989). Fabricación de helados. Zaragoza, España: Acribia. 